\title{
The anti-McCollough effect: the mystery remains
}

\author{
Bhavin R. Sheth
}

Published online: 26 July 2011

(C) Psychonomic Society, Inc. 2011

Felice Bedford (2011) makes some interesting points. The central thrust of her argument is that the anti-McCollough aftereffect (anti-ME) is not a new type of contingent aftereffect and, thus, can be explained by present theory. We have little doubt that present theory, including the author's own perceptual learning theory, will evolve to eventually explain all experimental findings. Nevertheless, a few salient points we raise below may lead one to pause before rushing to embrace fully Bedford's arguments.

One of Bedford's arguments is that a single orientationcolor pairing produces more ambiguous input than do the standard two orientation-color pairings used to produce a classical McCollough effect (ME). Although we have not induced with the standard two orientation-color pairs (Sheth \& Shimojo, 2008), we agree that a single orientation-color pairing is probably key to obtaining an anti-ME, mainly because a single pairing yields a weaker classical $\mathrm{ME}$, which, in turn, may be critical for the classical ME to be overpowered and subverted by the anti-ME during induction.

Bedford further argues that the contingency is ambiguous and the anti-ME may not be contingent on orientation at all. To the contrary, we argue that the anti-ME is contingent upon a number of features of the stimulus, including orientation and spatial frequency. A test compar-

B. R. Sheth $(\square)$

Department of Electrical and Computer Engineering,

University of Houston,

Houston, TX 77204-4005, USA

e-mail: brsheth@uh.edu

B. R. Sheth

Center for NeuroEngineering and Cognitive Science,

University of Houston,

Houston, TX 77204, USA ison of achromatic horizontal and vertical gratings of the same spatial frequency revealed a clear difference in their perceived color (Sheth \& Shimojo, 2008). That is to say, there is an orientation contingency, but the contingency is not exclusive to orientation alone.

Bedford contends that the single orientation-color pairing yields a degraded version of the effect one obtains with a homogeneous wide-field inducer, which is in line with her argument that inducer orientation is not important. It is worth noting that although Figs. 1 and 2 of the original article (Sheth \& Shimojo, 2008) suggest otherwise, there is no statistical difference in the size of the respective aftereffects obtained with homogeneous wide-field inducers versus horizontal inducers. Moreover, the slope - a proxy for sensitivity - of the postinduction psychometric curve in Fig. 1 of Sheth and Shomojo (2008), corresponding to the oriented, colored inducer, is clearly steeper than that in Fig. 2 of said reference, corresponding to the wide-field colored inducer. If the anti-ME is a degraded version of the effect with the wide-field inducer, as Bedford claims, the slope ought to be less, not more, steep. Moreover, we do not claim that the stimulus sequence we used in the original article is optimal and realizes the strongest possible anti$\mathrm{ME}$; we have not explored the parameter space as much as would like. In this regard, the strongest aftereffect that we have seen thus far is with a colored, oriented grating alternating with an orthogonally oriented achromatic onestronger numerically than that with alternating iso-oriented colored and achromatic inducers, as well as with alternating homogeneous wide-field and achromatic, oriented inducers. These experiments support the assertion that an anti-ME is contingent upon the orientation of the achromatic inducer, as we originally claimed.

The nearly half-century of research into the $\mathrm{ME}$ is replete with individuals who have attempted unsuccessfully 
to obtain interocular transfer, and only a fraction of their failures have been documented in publication. We count ourselves as one of them. Furthermore, although the ME did not transfer to the noninduced eye in our experiments, the anti-ME did (100\%). Even more interesting, even with stimulus parameters that we believed would typically yield an anti-ME, when the rare subject showed a classical ME (which, regrettably, we are unable to explain thus far), it did not transfer to the other eye (see Experiment 3 and Fig. 3 of Sheth \& Shimojo, 2008, for a more complete description). Conversely, with stimulus parameters that typically yield a classical ME, when the rare subject showed an anti-ME, the effect transferred $100 \%$ to the other eye (see the discussion on page 8 of Sheth \& Shimojo, 2008). This clearly argues for an effect that is different, at least in some ways, from the McCollough family of aftereffects. Interestingly, the AE obtained by alternately inducing with colored, wide-field and achromatic, oriented inducers did not transfer to the noninduced eye (unpublished observations). A minor point of clarification regarding our experiments is that the noninduced eye did not receive visual stimulation, diffuse or otherwise, and all our observers followed identical procedures.

Bedford's discussion about low- and high-level representations of aftereffects prompts us to report an exciting anecdotal observation. As Bedford states, the classic ME depends on retinal, not spatial, orientation and cannot be made dependent on spatial orientation either. That is to say, the ME flips with head rotation (the previously horizontal test gratings that appeared greenish now appear reddish after one's head is rotated $90^{\circ}$ ), and the anti-ME does not; that is, achromatic horizontal test gratings that appear greenish are unchanged even after head rotation. We agree with Bedford that this lends support to the assertion that the anti-ME is a higher level effect than is the ME. Shin
Shimojo, my coauthor on the original article, first noticed the dependence of the anti-ME on spatial, and not retinal, orientation, and we later anecdotally confirmed the observation on others. Unfortunately, we did not pursue this observation in a rigorous manner.

We do not state that the anti-ME is represented in a higher level area while the ME is simultaneously represented in a lower level area that can potentially be unmasked, as Bedford indirectly claims. An alternative claim, for which we have some basis (see Fig. 1c in Sheth \& Shimojo, 2008), is that with increasing induction duration, the perceptual balance shifts from a classical ME (or the lack of an AE in any direction) that was transiently represented in a lower level brain area to an anti-ME that is more permanently represented in a different, higher level brain area. The critical duration required for this transition may be dependent on observer.

In sum, Bedford raises some interesting points. The antiME remains a puzzling effect on top of another, not fully understood one - the ME. Our proposed interpretation is a first stab at explaining the somewhat perplexing data we obtained. We are open to the possibility — nay, probabilitythat the next generation of vision researchers will, with creative new experiments (e.g., by using binocularly rivalrous $\mathrm{ME}$ and anti-ME stimuli), refine, modify, or even outright change the present interpretation of our somewhat strange findings.

\section{References}

Bedford, F. L. Mystery of the anti-McCollough effect, Online First (tm), 21 June 2011; doi:10.3758/s13414-011-0163-1

Sheth, B. R., \& Shimojo, S. (2008). Adapting to an aftereffect. Journal of Vision, 8(3, Art. 29), 1-10. 